PROCEEDINGS OF THE

AMERICAN MATHEMATICAL SOCIETY

Volume 140, Number 7, July 2012, Pages 2215-2226

S 0002-9939(2011)11074-8

Article electronically published on October 24, 2011

\title{
F-BLOWUPS OF F-REGULAR SURFACE SINGULARITIES
}

\author{
NOBUO HARA
}

(Communicated by Lev Borisov)

\begin{abstract}
We prove that F-blowups of any F-regular surface singularity coincide with the minimal resolution.
\end{abstract}

In [Y1, Yasuda introduced the notion of the eth F-blowup, which is a canonical birational modification of varieties in characteristic $p>0$ defined as a flattening of the direct image of the structure sheaf by the eth iterate of the Frobenius morphism. Yasuda also showed the monotonicity of the sequence of F-blowups for F-pure singularities $\mathrm{Y2}$. Furthermore, it turns out that the eth F-blowup $\mathrm{FB}_{e}(X)$ of a surface singularity $X$ coincides with the minimal resolution for $e \gg 0$ if $X$ is a toric singularity [Y1, a tame quotient singularity [TY] or an F-rational double point HS.

The above results seem to suggest a connection of F-blowups with F-singularities such as F-purity and F-regularity, which are the concepts from commutative algebra defined via splitting of the Frobenius ring homomorphism in characteristic $p>0$; see e.g., $[\mathrm{HH},[\mathrm{Hu}$. Actually, toric and tame quotient singularities are F-regular as well as F-rational double points, while there are non-F-regular surface singularities whose F-blowups do not coincide with the minimal resolution [HS. Thus it is natural to ask if the F-blowups of F-singularities have particularly nice properties such as the minimal resolution.

In this paper, we give an affirmative answer to the above question:

Theorem 3.1. Let $(X, x)$ be an F-regular surface singularity defined over an algebraically closed field of characteristic $p>0$. Then the e-th F-blowup $\mathrm{FB}_{e}(X)$ of $X$ coincides with the minimal resolution of $X$ for $e \gg 0$.

To prove the theorem we first give a characterization of F-regular surface singularities in Theorem 2.1 A surface singularity $(X, x)$ in characteristic $p>0$ is F-regular if and only if its complete local ring $R=\hat{\mathcal{O}}_{X, x}$ is a pure subring of a regular local ring that is module-finite over $R$. It then follows that if $(X, x)$ is an F-regular surface singularity, then there are only finitely many isomorphism classes of indecomposable reflexive $R$-modules. Once we have established this property for $R$ (which is referred as "finite representation type"), we can directly use the (strong) F-regularity of $R$ to prove that the ring $R^{1 / p^{e}}$ of $p^{e}$ th roots of $R$ (or the eth Frobenius direct image $F_{*}^{e} \mathcal{O}_{X}$ of $R=\mathcal{O}_{X}$ ) contains all indecomposable reflexive $R=\mathcal{O}_{X}$-modules as its direct summands for $e \gg 0$ (Theorem 3.3). Thanks to this

Received by the editors December 23, 2010 and, in revised form, February 14, 2011.

2010 Mathematics Subject Classification. Primary 13A35, 14 B05.

The author is partially supported by Grant-in-Aid for Scientific Research, JSPS.

(C)2011 American Mathematical Society

Reverts to public domain 28 years from publication 
key theorem, we are able to prove Theorem 3.1 by the aid of Wunram's result W] on the correspondence between "special" reflexive modules and exceptional curves of the minimal resolution.

There are some similarities between F-regular surface singularities in characteristic $p>0$ and quotient surface singularities in characteristic zero. The local rings of both singularities are pure subrings of a module-finite regular local ring. Also, Corollary 3.6] is considered an analogue of Ishii's result [I] that the $G$-Hilbert scheme of a complex quotient surface singularity is the minimal resolution. These analogies seem to suggest that for surface singularities, F-regularity is the right notion in characteristic $p>0$ that corresponds to quotient singularity in characteristic zero.

\section{Preliminaries}

F-regularity. The notion of F-regularity was first defined in terms of "tight closure" by Hochster and Huneke. To avoid technicality involving tight closure, we will adopt another version of F-regularity called strong F-regularity, which is known to coincide with the one defined via tight closure for $\mathbb{Q}$-Gorenstein rings (and in particular in dimension two). Although it is not known whether or not these two variants of F-regularity coincide in general, we often say just "F-regular" to mean "strongly F-regular," since we mostly work on surface singularities in this paper.

Definition $1.1([\mathrm{HH}])$. Let $R$ be an integral domain of characteristic $p>0$ which is F-finite (i.e., the inclusion map $R \hookrightarrow R^{1 / p}$ is module-finite). We say that $R$ is strongly F-regular if for every nonzero element $c \in R$, there exists a power $q=p^{e}$ such that the inclusion map $c^{1 / q} R \hookrightarrow R^{1 / q}$ splits as an $R$-module homomorphism.

1.2. We use the following basic properties of (strongly) F-regular rings $[\mathrm{HH}]$ :

(1) Regular rings are F-regular.

(2) Pure subring: 11 of an F-regular ring are F-regular.

(3) In particular, if $R$ is a subring of an F-regular ring $S$ such that $R$ is a direct summand of $S$ as an $R$-module, then $R$ is F-regular.

1.3. F-regular vs. splinter. One of the important ring-theoretic properties of $\mathrm{F}$ regularity is that an F-regular domain $R$ of characteristic $p>0$ is a direct summand as an $R$-module of every module-finite extension ring; cf. [ $\mathrm{Hu}$, Theorem 1.7]. Rings having this property are called splinters. It is proved by Singh $[\mathbf{S i}$ that this splinter property characterizes F-regularity for $\mathbb{Q}$-Gorenstein rings in characteristic $p>0$.

For the reader's convenience, we give a brief proof to an a priori weaker statement which will be used later.

Proposition 1.4. Let $R$ be an F-finite domain of characteristic $p>0$. If $R$ is strongly F-regular, then $R$ is a splinter.

Proof. Let $R \subset S$ be any module-finite extension. Then there exists $\phi \in \operatorname{Hom}_{R}(S, R)$ such that $c:=\phi(1)$ is a nonzero element of $R$. Then by the strong F-regularity of $R$, there exist a power $q=p^{e}$ of $p$ and $\psi \in \operatorname{Hom}_{R}\left(R^{1 / q}, R\right)$ such that $\psi\left(c^{1 / q}\right)=1$.

\footnotetext{
${ }^{1}$ A ring extension $R \subseteq S$ is said to be pure if for all $R$-modules $M$, the induced $R$-module homomorphism $M=R \otimes_{R} M \rightarrow S \otimes_{R} M$ is injective. When $S$ is module-finite over $R$, this is equivalent to the condition that the map $R \hookrightarrow S$ splits as an $R$-module homomorphism.
} 
Let $\phi^{1 / q} \in \operatorname{Hom}_{R^{1 / q}}\left(S^{1 / q}, R^{1 / q}\right)$ be the map corresponding to $\phi$. Then $\psi \circ \phi^{1 / q} \in$ $\operatorname{Hom}_{R}\left(S^{1 / q}, R\right)$ gives a splitting of $R \hookrightarrow R^{1 / q} \hookrightarrow S^{1 / q}$. Since this ring extension factors through $S$, the extension $R \hookrightarrow S$ also splits, via which $R$ is a direct summand of $S$ as an $R$-module.

1.5. F-regular vs. log terminal $([\mathrm{H}],[\mathrm{HW}])$. F-regularity is closely related to $\log$ terminal singularity. Namely, we have the implication

$$
\text { F-regular and } \mathbb{Q} \text {-Gorenstein } \Rightarrow \text { log terminal singularity }
$$

in arbitrary characteristic. The converse of this implication also holds in characteristic $p \gg 0$. In dimension two, F-regular rings are always $\mathbb{Q}$-Gorenstein, so that the implication "F-regular $\Rightarrow \log$ terminal singularity" holds without assuming the $\mathbb{Q}$-Gorensteinness. Let us discuss the case of surface singularities more in detail.

Let $(X, x)$ be a normal surface singularity defined over an algebraically closed field $k$ and let $\mu: \widetilde{X} \rightarrow X$ be the minimal resolution with irreducible exceptional curves $E_{1}, \ldots, E_{s}$. The numerical anti-discrepancy divisor $\Delta$ of $\mu$ is defined to be the $\mu$-exceptional $\mathbb{Q}$-divisor on $\widetilde{X}$ such that $\Delta E_{i}=-K_{\tilde{X}} E_{i}$ for $1 \leq i \leq s$. Note that $\Delta$ is an effective divisor by the minimality of $\mu$. The singularity $(X, x)$ is said to be $\log$ terminal if the integral part of $\Delta$ is zero, i.e., $\lfloor\Delta\rfloor=02$ Since this is a numerical condition depending only on the intersection matrix $\left(E_{i} E_{j}\right)_{1 \leq i, j \leq s}$ of the exceptional curves and their genera, log terminal surface singularities are classified in terms of the weighted dual graph $\Gamma$ associated to the exceptional set $E=\bigcup_{i=1}^{s} E_{i}$. In particular, if $(X, x)$ is $\log$ terminal, then $\Gamma$ is a chain or a star-shaped graph with three branches, and each $E_{i} \cong \mathbb{P}^{1}$. In the latter case, we associate to $\Gamma$ a triple $\left(d_{1}, d_{2}, d_{3}\right)$ consisting of the absolute values of the determinants of the intersection matrices of the three branches. In this notation, $(X, x)$ is a log terminal singularity if and only if $E_{i} \cong \mathbb{P}^{1}$ for $1 \leq i \leq s$ and either one of the following holds:

(A) $\Gamma$ is a chain;

(D) $\quad \Gamma$ is star-shaped with $\left(d_{1}, d_{2}, d_{3}\right)=(2,2, n)$, where $n \geq 2$;

$\left(E_{6}\right) \quad \Gamma$ is star-shaped with $\left(d_{1}, d_{2}, d_{3}\right)=(2,3,3)$;

$\left(E_{7}\right) \quad \Gamma$ is star-shaped with $\left(d_{1}, d_{2}, d_{3}\right)=(2,3,4)$;

$\left(E_{8}\right) \quad \Gamma$ is star-shaped with $\left(d_{1}, d_{2}, d_{3}\right)=(2,3,5)$.

The graphs appearing in this classification are exactly the same as the graphs of quotient surface singularities in characteristic zero, but a log terminal surface singularity in characteristic $p>0$ is not a quotient singularity in general.

The classification of F-regular surface singularities obtained in $[\underline{H}]$ is as follows: $(X, x)$ is F-regular if and only if the type of the singularity and characteristic $p>0$ is either one of the following:

(1) type $A$ (possibly smooth), $p$ is arbitrary;

(2) type $D, p \neq 2$;

(3) type $E_{6}$ or $E_{7}, p>3$;

(4) type $E_{8}, p>5$.

In particular, log terminal surface singularities in characteristic $p>5$ are F-regular.

F-blowups. In what follows, we work over an algebraically closed field $k$ of characteristic $p>0$. For a variety $X$ over $k$ and an integer $e \geq 0$, let $F_{\text {rel }}^{e}: X^{(e)} \rightarrow X$ be

\footnotetext{
${ }^{2}$ This condition implies that $(X, x)$ is a rational surface singularity from which the $\mathbb{Q}$ Gorensteinness automatically follows.
} 
the $e$-times iterated $k$-linear Frobenius morphism. We identify this relative Frobenius with the morphism $X^{1 / p^{e}}:=\operatorname{Spec}_{X} \mathcal{O}_{X}^{1 / p^{e}} \rightarrow X$ induced by the inclusion map $\mathcal{O}_{X} \hookrightarrow \mathcal{O}_{X}^{1 / p^{e}}$ into the ring of $p^{e}$ th roots. (We also abuse the absolute and relative Frobenius, since it is harmless under our assumption that $k$ is algebraically closed.)

Now, the fiber $\left(F_{\text {rel }}^{e}\right)^{-1}(x)$ of the $e$-times iterated Frobenius over a smooth (closed) point $x \in X$ is a fat point of $X^{(e)}$ of length $p^{n e}$, where $n=\operatorname{dim} X$. Thus, it is regarded as a reduced point of the Hilbert scheme $\operatorname{Hilb}_{p^{n e}}\left(X^{(e)}\right)$ of zero-dimensional subschemes of $X^{(e)}$ of length $p^{n e}$.

Definition 1.6 (Yasuda Y1]). Let $X$ be a variety of $\operatorname{dim} X=n$ over $k$. The $e$ th F-blowup $\mathrm{FB}_{e}(X)$ of $X$ is defined to be the closure of the subset

$$
\left\{\left(F_{\text {rel }}^{e}\right)^{-1}(x) \mid x \in X(k) \text { smooth }\right\} \subseteq \operatorname{Hilb}_{p^{n e}}\left(X^{(e)}\right) .
$$

It is proved in [Y1] that it is birational and projective over $X$. Indeed, $\mathrm{FB}_{e}(X)$ is isomorphic to the irreducible component $\operatorname{Hilb}_{p^{n e}}\left(X^{(e)} / X\right)^{\circ}$ of the relative Hilbert scheme $\operatorname{Hilb}_{p^{n e}}\left(X^{(e)} / X\right) \cong \operatorname{Hilb}_{p^{n e}}\left(X^{1 / p^{e}} / X\right)$ that dominates $X$. We denote the associated structure morphism by $\varphi=\varphi_{e}: \mathrm{FB}_{e}(X) \rightarrow X$.

By definition, $\varphi: Z=\mathrm{FB}_{e}(X) \rightarrow X$ is a birational projective morphism such that the torsion-free pullback $\varphi^{\star} \mathcal{O}_{X}^{1 / p^{e}}:=\varphi^{*} \mathcal{O}_{X}^{1 / p^{e}} /$ torsion is a flat (equivalently, locally free) $\mathcal{O}_{Z}$-algebra of $\operatorname{rank} p^{n e}$, and $\mathrm{FB}_{e}(X)$ is universal with respect to this property.

Our main concern is the following question in the surface case $n=2$.

Question. Let $(X, x)$ be a normal surface singularity over $k$ and let $\mu: \widetilde{X} \rightarrow X$ be the minimal resolution. When is $\mathrm{FB}_{e}(X)$ equal to the minimal resolution $\tilde{X}$ ?

It is proved that $\mathrm{FB}_{e}(X)=\widetilde{X}$ for $e \gg 0$ if $(X, x)$ is either a toric singularity [Y1], a tame quotient singularity [TY, or an F-regular double point $[\mathrm{HS}$. We note that $\mathcal{O}_{X, x}$ is F-regular in all of the above three cases. When $X=S / G$ is a tame quotient of smooth $S$ by a finite group $G$, the essential part is to prove the isomorphism $\mathrm{FB}_{e}(X) \cong \operatorname{Hilb}^{G}(S)$ of the F-blowup with the $G$-Hilbert scheme [Y1, [TY], since $G$-Hilb of a tame quotient surface singularity is the minimal resolution by a work of Ishii [I]. In general, an F-regular surface singularity in low characteristic $p>0$ is not a quotient singularity, so the group $G$ and $G$-Hilb are not available. However, we can use the rationality of F-regular surface singularities.

A few lemmata on rational surface singularities. Throughout the remainder of this section, we assume that $(X, x)$ is a rational surface singularity and $\mu: \widetilde{X} \rightarrow X$ is any resolution of the singularity.

It is known that if $M$ is a finitely generated reflexive $\mathcal{O}_{X}$-module, then its torsionfree pullback $\widetilde{M}=\mu^{\star} M$ is a $\mu$-generated locally free $\mathcal{O}_{\widetilde{X}}$-module such that $\mu_{*} \widetilde{M}=$ $M$ and $R^{1} \mu_{*} \widetilde{M}=0$; see e.g., $[\mathrm{AV}]$. Note that $R^{1} \mu_{*} \mathcal{F}=0$ for any $\mu$-generated coherent sheaf on $\tilde{X}$. This is an easy consequence of the rationality of the singularity $(X, x)$ and the $\mu$-generation of $\mathcal{F}$, which gives rise to a surjection $\mathcal{O}_{\widetilde{X}}^{\oplus m} \rightarrow \mathcal{F}$.

In particular, since $\mathcal{O}_{X}^{1 / q}$ is a reflexive $\mathcal{O}_{X}$-module of rank $q^{2}$, we have the following.

Lemma 1.7 ([S, Corollary 4.6]). The e-th F-blowup $\mathrm{FB}_{e}(X)$ of a rational surface singularity $(X, x)$ is dominated by the minimal resolution for all $e \geq 0$. 
Lemma 1.8. Let $(X, x)$ be a rational surface singularity and let $\mu: \widetilde{X} \rightarrow X$ be any resolution of the singularity. If $M$ is a reflexive $\mathcal{O}_{X}$-module of rank $r$, then the natural map

is surjective.

$$
\bigwedge^{r} M \rightarrow \mu_{*}\left(\operatorname{det} \mu^{\star} M\right)
$$

Proof. Let $\widetilde{M}=\mu^{\star} M$. It is sufficient to show that the natural map $M^{\otimes i} \rightarrow$ $\mu_{*}\left(\bigwedge^{i} \widetilde{M}\right)$ is surjective for all $i=1, \ldots, r$. We factorize this map into two maps, $\alpha_{i}: M^{\otimes i}=\left(\mu_{*} \widetilde{M}\right)^{\otimes i} \rightarrow \mu_{*}\left(\widetilde{M}^{\otimes i}\right)$ and $\beta_{i}: \mu_{*}\left(\widetilde{M}^{\otimes i}\right) \rightarrow \mu_{*}\left(\bigwedge^{i} \widetilde{M}\right)$, and prove that $\alpha_{i}$ and $\beta_{i}$ are surjective in two steps.

Step 1 . We know that $\alpha_{1}$ is surjective. Therefore, let $i \geq 2$ and prove the surjectivity of $\alpha_{i}$ by induction on $i$. Let $\mathcal{T}$ be the torsion part of the $\mathcal{O}_{\widetilde{X}^{-}}$-module $f^{*} M$ so that $\widetilde{M}=f^{*} M / \mathcal{T}$. We fix generators $s_{1}, \ldots, s_{m}$ of $M=f_{*} \frac{\widetilde{X}_{M}}{\text { and consider }}$ the associated surjection $\sigma: \mathcal{O}_{X}^{\oplus m} \rightarrow M$. Then we have the following two exact sequences:

$$
\begin{gathered}
0 \rightarrow \mathcal{S} \rightarrow \mathcal{O}_{\widetilde{X}}^{\oplus m} \stackrel{\mu^{\star} \sigma}{\longrightarrow} \widetilde{M} \rightarrow 0, \\
0 \rightarrow \mu^{\star} \operatorname{Ker}(\sigma) \rightarrow \mathcal{S} \rightarrow \mathcal{T} \rightarrow 0 .
\end{gathered}
$$

The long exact sequence of $(1) \otimes \widetilde{M}^{\otimes i-1}$ gives

$$
\mu_{*}\left(\widetilde{M}^{\otimes i-1}\right)^{\oplus m} \stackrel{\sigma^{\prime}}{\longrightarrow} \mu_{*}\left(\widetilde{M}^{\otimes i}\right) \rightarrow R^{1} \mu_{*}\left(\mathcal{S} \otimes \widetilde{M}^{\otimes i-1}\right) \rightarrow 0,
$$

where the image of the map $\sigma^{\prime}=\mu_{*}\left(\mu^{\star} \sigma \otimes \widetilde{M}^{\otimes i-1}\right)$ coincides with the image of the map $\alpha_{i}: M^{\otimes i} \rightarrow \mu_{*}\left(\widetilde{M}^{\otimes i}\right)$ by induction hypothesis. Hence the surjectivity of $\alpha_{i}$ would follow if $R^{1} \mu_{*}\left(\mathcal{S} \otimes \widetilde{M}^{\otimes i-1}\right)=0$. It then suffices to show that $R^{1} \mu_{*} \mathcal{T}=0$, because $\widetilde{M}^{\otimes i-1}$ is $\mu$-generated and $R^{1} \mu_{*}\left(\mathcal{S} \otimes \widetilde{M}^{\otimes i-1}\right)=R^{1} \mu_{*}\left(\mathcal{T} \otimes \widetilde{M}^{\otimes i-1}\right)$ by (2). To see this we consider the long exact sequence of $0 \rightarrow \mathcal{T} \rightarrow \mu^{*} M \rightarrow \widetilde{M} \rightarrow 0$ :

$$
\mu_{*} \mu^{*} M \rightarrow \mu_{*} \widetilde{M} \rightarrow R^{1} \mu_{*} \mathcal{T} \rightarrow R^{1} \mu_{*} \mu^{*} M
$$

Here the map $\mu_{*} \mu^{*} M \rightarrow \mu_{*} \widetilde{M}$ on the left is surjective since the identity map of $M$ is factorized as $M \rightarrow \mu_{*} \mu^{*} M \rightarrow \mu_{*} \widetilde{M}=M$, and $R^{1} \mu_{*} \mu^{*} M=0$ since $\mu^{*} M$ is $\mu$-generated. Thus $R^{1} \mu_{*} \mathcal{T}=0$, as required.

Step 2. Let $I_{i}=\operatorname{Ker}\left(\widetilde{M}^{\otimes i} \rightarrow \bigwedge^{i} \widetilde{M}\right)$. If $I_{i}$ is $\mu$-generated, then $R^{1} \mu_{*} I_{i}=0$ so that the required surjectivity of $\beta_{i}$ follows from the exact sequence

$$
\mu_{*}\left(\widetilde{M}^{\otimes i}\right) \rightarrow \mu_{*}\left(\bigwedge^{i} \widetilde{M}\right) \rightarrow R^{1} \mu_{*} I_{i}=0 .
$$

We assume that $X$ is affine and deduce the global generation of $I_{2}$ from that of $\widetilde{M}$. Recall that $I_{2}$ is generated by local sections of the form $x \otimes x$, where $x$ is any local section of $\widetilde{M}$. We fix global sections $s_{1}, \ldots, s_{m} \in M$ that generate $\widetilde{M}$ and write $x=\sum_{i=1}^{m} a_{i} s_{i}$ with local regular functions $a_{1}, \ldots, a_{m}$ on $\widetilde{X}$. Then

$$
x \otimes x=\sum_{1 \leq i, j \leq m} a_{i} a_{j}\left(s_{i} \otimes s_{j}\right)=\sum_{i=1}^{m} a_{i}^{2}\left(s_{i} \otimes s_{i}\right)+\sum_{1 \leq i<j \leq m} a_{i} a_{j}\left(s_{i} \otimes s_{j}+s_{j} \otimes s_{i}\right),
$$

from which we see that $I_{2}$ is generated by its global sections $s_{i} \otimes s_{i}$ and $s_{i} \otimes s_{j}+s_{j} \otimes s_{i}$ for $1 \leq i, j \leq m$. 
Now the global generation of $I_{i}$ for any $i \geq 0$ follows from that of $I_{2}$ and $\widetilde{M}$, since $I=\bigoplus_{i \geq 0} I_{i}$ is the two-sided ideal of the tensor algebra $T(\widetilde{M})=\bigoplus_{i \geq 0} \widetilde{M}^{\otimes i}$ generated by all local sections $x \otimes x$ of $I_{2}$.

\section{Covering of F-Regular surface Singularities}

This section is devoted to proving the following characterization of F-regular surface singularities.

Theorem 2.1. Let $(R, \mathfrak{m})$ be a two-dimensional complete local ring with algebraically closed coefficient field $k=R / \mathfrak{m}$ of characteristic $p>0$. Then $R$ is $F$ regular if and only if there exists a module-finite extension of local rings $R \subset k[[t, u]]$ via which $R$ is a pure subring of the regular local ring $k[[t, u]]$.

Proof. The sufficiency is clear from subsection 1.2, so we prove the necessity. Let $X=\operatorname{Spec} R$ and let $\mu: \widetilde{X} \rightarrow X$ be the minimal resolution of the closed point $x=\mathfrak{m} \in X$. If $R$ is F-regular, then $(X, x)$ is a log terminal singularity and either one of cases (1)-(4) in subsection 1.5 occurs. First suppose that $p \neq 2,3$. Then there exists a finite covering $(Y, y) \rightarrow(X, x)$ with $(Y, y)$ smooth or a rational double point by Kawamata $[\mathrm{K}$, while $(Y, y)$ is covered by a smooth surface germ $(S, o)$ by Artin [Ar2. Hence we have a module-finite extension $R=\mathcal{O}_{X, x} \subset \mathcal{O}_{S, o}=k[[t, u]]$, via which $R$ is a pure subring of $k[[t, u]]$ by Proposition [1.4 Thus the assertion follows in cases (3) and (4) of subsection 1.5. We consider the remaining cases (1) and (2) separately.

Case (1). Assume that $(X, x)$ is a log terminal singularity of type $A$. In this case it follows that $(X, x)$ is a toric singularity. We shall sketch the proof given in BHPV, III.5], since it seems less known in characteristic $p>03$ Let $E=\bigcup_{i=1}^{s} E_{i}$ be the exceptional set of $\mu$ with irreducible components $E_{i} \cong \mathbb{P}^{1}$ and set $a_{i}=-E_{i}^{2} \geq 2$. By the completeness of $R$ we can choose divisors $B_{0}$ and $B_{s+1}$ on $\widetilde{X}$ that intersect $E$ transversally so that $B_{0}, B_{s+1}$ and the $E_{i}$ 's are arranged as follows:

$$
B_{0}-E_{1}-E_{2}-\cdots-E_{r}-B_{s+1} \text {. }
$$

Let $n, q$ be the coprime integers with $0<q<n$ determined by the continued fraction

$$
\frac{n}{q}=a_{1}-\frac{1}{a_{2}-\frac{1}{\ddots}-\frac{1}{a_{s}}}
$$

Using a recursion formula involving $a_{i}$ 's as in BHPV, III.5], we can find $\mu$-numerically trivial effective Cartier divisors $Z=n B_{0}+q E_{1}+\left(\right.$ terms of $\left.E_{2}, \ldots, E_{s}\right)$ and $Z^{\prime}=E_{1}+\left(\right.$ terms of $\left.E_{2}, \ldots, E_{s}, B_{s+1}\right)$ on $\widetilde{X}$ such that $\frac{1}{n}\left(Z+(n-q) Z^{\prime}\right)$ is Cartier. Since $\mu$-numerical equivalence of Cartier divisors on $\widetilde{X}$ is $\mu$-linear equivalence by the rationality of $(X, x)$ [Ar1, there exist regular functions $f, g, h$ on $\widetilde{X}$ such that $Z=$ $\operatorname{div}_{\tilde{X}}(f), Z^{\prime}=\operatorname{div}_{\widetilde{X}}(g)$ and $h^{n}=f g^{n-q}$. Thus $\widetilde{X}$ is mapped into the hypersurface $W$ defined by $z^{n}=x y^{n-q}$.

\footnotetext{
${ }^{3}$ In characteristic zero, this is a consequence of the well-known "tautness" of quotient surface singularities. On the other hand, the argument in BHPV] III.5] goes through also in characteristic $p>0$, although it is stated for complex singularities.
} 
Consider the ring homomorphism $k[[x, y]] \rightarrow R=H^{0}\left(\widetilde{X}, \mathcal{O}_{\tilde{X}}\right)$ sending $x, y$ to $f, g$, respectively, and let

$$
\rho: \tilde{X} \stackrel{\mu}{\longrightarrow} X \stackrel{\gamma}{\longrightarrow} S=\operatorname{Spec} k[[x, y]]
$$

be the corresponding morphisms. Since the divisors $Z=\operatorname{div}_{\widetilde{X}}(f)$ and $Z^{\prime}=\operatorname{div}_{\widetilde{X}}(g)$ intersect exactly on $E$, we have $\rho^{-1}(o)=E$. Hence $(f, g) \subset R$ is an ideal of finite colength so that $\gamma$ is a finite morphism. Since $\gamma$ factors through the normalization $\widetilde{W}$ of $W$, which is an $n$-fold covering of $S$, we would obtain $X \cong \widetilde{W}$ as soon as we know that $\operatorname{deg} \gamma=n$. To see this, let $C_{0} \subset X$ and $L_{0}=\operatorname{div}_{S}(x) \subset S$ be the images of the curve $B_{0} \subset \widetilde{X}$, and consider the module-finite extension of discrete valuation rings $\mathcal{O}_{S, L_{0}} \hookrightarrow \mathcal{O}_{X, C_{0}} \cong \mathcal{O}_{\tilde{X}, B_{0}}$, via which the regular parameter $x$ of $\mathcal{O}_{S, L_{0}}$ maps to an element $f$ of order $n$ in $\mathcal{O}_{\widetilde{X}, B_{0}}$. Also, it induces an isomorphism $k((y)) \cong k((g))$ of the residue fields, since $g$ is part of a regular system of parameters at $B_{0} \cap E_{1}$. Hence $\mathcal{O}_{\widetilde{X}, B_{0}}$ is a free $\mathcal{O}_{S, L_{0}}$-module of rank $n$ so that $\operatorname{deg} \gamma=n$. Consequently, $R$ is isomorphic to the normalization of $\mathcal{O}_{W, o}=k[[x, y, z]] /\left(z^{n}-\right.$ $\left.x y^{n-q}\right) \cong k\left[\left[t^{n}, u^{n}, t u^{n-q}\right]\right] \subset k[[t, u]]$, from which we obtain a desired extension $R \subset k[[t, u]]$.

Case (2). Assume that $(X, x)$ is a log terminal singularity of type $D$ and $p \neq 2$. Let the exceptional curves $E_{1}, \ldots, E_{s}$ of $\mu$ be indexed as

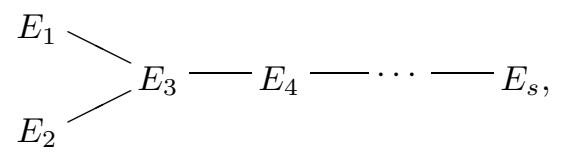

where $E_{1}^{2}=E_{2}^{2}=-2$. Since $R$ is complete, we can choose effective divisors $D_{i}$ on $\widetilde{X}$ such that $D_{i} E_{j}=\delta_{i j}$. Let $B=E_{1}+E_{2}$ and $D=D_{1}+D_{2}-D_{3}$. Then $B E_{i}=-2 D E_{i}$ for $1 \leq i \leq s$ so that $B \sim-2 D$ since $(X, x)$ is a rational singularity Ar1. The map $\mathcal{O}_{\widetilde{X}}(D)^{\otimes 2} \cong \mathcal{O}_{\widetilde{X}}(-B) \hookrightarrow \mathcal{O}_{\tilde{X}}$ gives rise to an $\mathcal{O}_{\widetilde{X}}$-algebra structure on $\mathcal{A}=\mathcal{O}_{\tilde{X}} \oplus \mathcal{O}_{\tilde{X}}(D)$, and we have a double cover $\widetilde{\pi}: \widetilde{Y}=\operatorname{Spec}_{\widetilde{X}} \mathcal{A} \rightarrow \widetilde{X}$ branched over $B=E_{1}+E_{2}$. We note that $\widetilde{Y}$ is smooth since $B$ is smooth, and $\widetilde{\pi}$ induces a double cover $\pi:(Y, y) \rightarrow(X, x)$ that fits in the following commutative diagram:

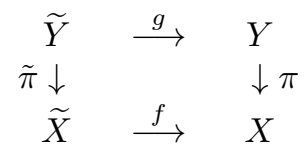

Here $g: \tilde{Y} \rightarrow Y$ is a proper birational morphism, and it is easy to see that its exceptional set $g^{-1}(y)=\bigcup F_{j}$ consists of smooth rational curves $F_{1}, \ldots, F_{2 s-3}$ arranged as follows:

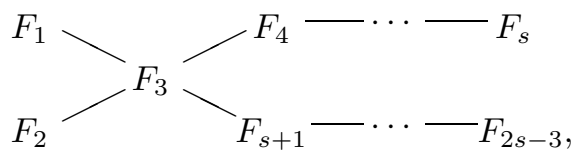


where $F_{1}^{2}=F_{2}^{2}=-1, F_{3}^{2}=2 E_{3}^{2}$ and $F_{i}^{2}=F_{s-3+i}^{2}=E_{i}^{2}$ for $4 \leq i \leq s$. Contracting the $(-1)$-curves $F_{1}$ and $F_{2}$, we obtain a chain of $2 s-5$ rational curves, which contracts to $(Y, y)$. Thus $(Y, y)$ is of type $A$, which admits a covering $(S, o) \rightarrow(Y, y)$ from a smooth surface germ as we have seen in Case (1). Composing this with the double cover $\pi$, we obtain a desired covering $(S, o) \rightarrow(X, x)$.

It follows from Theorem 2.1 that a two-dimensional F-regular local ring $R=$ $\mathcal{O}_{X, x}$ has finite representation type, that is, there are only finitely many isomorphism classes of indecomposable maximal Cohen-Macaulay $R$-modules. (Note that maximal Cohen-Macaulay modules over a two-dimensional ring are the same as the reflexive modules.)

Corollary 2.2 (cf. Aus, Proposition 2.1]). Let (R, m) be a two-dimensional complete local ring with algebraically closed coefficient field $k=R / \mathfrak{m}$ of characteristic $p>0$. If $R$ is F-regular, then $R$ has finite representation type.

Proof. By Theorem 2.1, $R$ is a pure subring of a complete regular local ring $k[[x, y]]$ that is module-finite over $R$. We abuse the notation to denote $S=k[[x, y]]$.

Let $M$ be any indecomposable reflexive $R$-module and let $M^{\vee}=\operatorname{Hom}_{R}(M, R)$ be its $R$-dual. By the purity of the ring extension $R \subset S$, the monomorphism of $R$ modules $\operatorname{Hom}_{R}\left(M^{\vee}, R\right) \rightarrow \operatorname{Hom}_{R}\left(M^{\vee}, S\right)$ splits. Since $\operatorname{Hom}_{R}\left(M^{\vee}, S\right)$ is a reflexive module over a two-dimensional regular local $\operatorname{ring} S$, it is a free $S$-module so that $M=\operatorname{Hom}_{R}\left(M^{\vee}, R\right)$ is a direct summand as an $R$-module of a finite sum $S^{\oplus n}$. We note that the category of $R$-modules is a Krull-Schmidt category because $R$ is complete. Hence the fact that $M$ is indecomposable and a direct summand of $S^{\oplus n}$ implies that $M$ is a direct summand of $S$.

It follows that $S$ is a full $R$-module; that is, every indecomposable reflexive module is isomorphic to a direct summand of the $R$-module $S$. Since $S$ is modulefinite over $R$, the conclusion follows.

Question. We may ask if Theorem 2.1 and Corollary 2.2 remain true in the absence of F-regularity. Let $(X, x)$ be any log terminal surface singularity. Then:

(1) Does there exist a finite covering $S \rightarrow X$ from a smooth surface germ $(S, o)$ ?

(2) Does $R=\mathcal{O}_{X, x}$ have finite representation type?

It is known that (1) and (2) are affirmative for rational double points $\mathrm{Ar2}$, AV].

\section{F-Blowups of F-REgular SURface Singularities}

Throughout this section we work under the following notation: Let $(X, x)$ be a normal surface singularity defined over an algebraically closed field of characteristic $p>0$. Since our problem is local, we will presumably put $X=\operatorname{Spec} R$, where $R=\mathcal{O}_{X}=\mathcal{O}_{X, x}$.

The purpose of this section is to prove the following theorem conjectured in [HS].

Theorem 3.1. If $(X, x)$ is an F-regular surface singularity, then its e-th F-blowup $\mathrm{FB}_{e}(X)$ coincides with the minimal resolution of $X$ for $e \gg 0$.

This generalizes Proposition 4.9 of [HS], which is proved for (F-)rational double points only. This earlier result is based on an explicit description of the McKay correspondence involving a covering of the rational double point by a smooth surface ([AV], GSV] $)$. We give here a simpler approach that directly uses strong F-regularity without referring to any explicit description of a covering. 
Proof. To begin with, let $\hat{R}$ be the $\mathfrak{m}_{x^{-}}$adic completion of $R$ and let $\hat{X}=\operatorname{Spec} \hat{R}$. Then the F-regularity of $R$ inherits to $\hat{R}$, and $\operatorname{FB}_{e}(\hat{X}) \cong \mathrm{FB}_{e}(X) \times_{X} \hat{X}$ by $[\mathrm{Y} 1$, Proposition 2.11]. Since $(X, x)$ is an isolated singularity, we may assume without loss of generality that $R$ is a complete local ring to prove Theorem 3.1.

Thanks to Corollary 2.2, the F-regularity of $R$ implies that it has finite representation type. Let $M_{1}, \ldots, M_{n}$ be the (isomorphism classes of) indecomposable reflexive $\mathcal{O}_{X, x}$-modules.

The next few results may hold in arbitrary dimension. We first show an easy lemma.

Lemma 3.2. Let $R$ be an integral domain and let $M_{1}, \ldots, M_{n}$ be finitely generated torsion-free $R$-modules with $\operatorname{rank} M_{i}=r_{i}$. Then there is a non-zero element $c \in R$ such that for all $i=1, \ldots, n$, the multiplication map by $c: M_{i} \rightarrow M_{i} ; m \mapsto \mathrm{cm}$, factors through the free module of rank $r_{i}$ as $M_{i} \rightarrow R^{\oplus r_{i}} \rightarrow M_{i}$.

Proof. It is easy to see that we can choose $0 \neq c_{i} \in R$ such that the multiplication by $c_{i}$ factors as $M_{i} \rightarrow R^{\oplus r_{i}} \rightarrow M_{i}$. Then the product $c=c_{1} \cdots c_{n}$ satisfies the required property.

The following is our key technical result; cf. Corollary 2.2.

Theorem 3.3. Let $R=\mathcal{O}_{X}$ be a strongly F-regular complete local ring with only finitely many isomorphism classes of indecomposable reflexive $R$-modules $M_{1}, \ldots$, $M_{n}$. Then the $R$-module $R^{1 / p^{e}} \cong F_{*}^{e} \mathcal{O}_{X}$ is full for $e \gg 0$, that is, $M_{i}$ is isomorphic to a direct summand as an $R$-module of $R^{1 / p^{e}}$.

Proof. Choose $c$ as in Lemma 3.2 and let $M_{i}^{\vee}=\operatorname{Hom}_{R}\left(M_{i}, R\right)$ be the $R$-dual of $M_{i}$. Then, since $R$ is strongly F-regular, there exists $q=p^{e}$ such that the monomorphism of $R$-modules

$$
M_{i}=\operatorname{Hom}_{R}\left(M_{i}^{\vee}, R\right) \stackrel{c^{1 / q}}{\longrightarrow} \operatorname{Hom}_{R}\left(M_{i}^{\vee}, R^{1 / q}\right)
$$

splits 4 Since $\operatorname{Hom}_{R}\left(M_{i}^{\vee}, R^{1 / q}\right) \cong \operatorname{Hom}_{\mathcal{O}_{X}}\left(M_{i}^{\vee}, F_{*}^{e} \mathcal{O}_{X}\right)$ is a reflexive module over $R^{1 / q} \cong F_{*}^{e} \mathcal{O}_{X}$, it is isomorphic to a direct sum of copies of $F_{*}^{e} M_{i}(i=1, \ldots, n)$ : $\operatorname{Hom}_{R}\left(M_{i}^{\vee}, R^{1 / q}\right) \cong \bigoplus_{j=1}^{n} F_{*}^{e} M_{j}^{\oplus a_{i j}}$. Thus by Lemma 3.2, the multiplication map by $c^{1 / q}=F_{*}^{e}(c)$ on $\operatorname{Hom}_{R}\left(M_{i}^{\vee}, R^{1 / q}\right) \cong \operatorname{Hom}_{\mathcal{O}_{X}}\left(M_{i}^{\vee}, F_{*}^{e} \mathcal{O}_{X}\right)$ factors through the free module $\left(R^{1 / q}\right)^{\oplus r_{i}} \cong F_{*}^{e} \mathcal{O}_{X}^{\oplus r_{i}}$, where $r_{i}=\sum_{j=1}^{n} a_{i j} r_{j}$. Hence the monomorphism (3) is factorized as

$$
M_{i} \rightarrow \operatorname{Hom}_{R}\left(M_{i}^{\vee}, R^{1 / q}\right) \rightarrow\left(R^{1 / q}\right)^{\oplus r_{i}} \rightarrow \operatorname{Hom}_{R}\left(M_{i}^{\vee}, R^{1 / q}\right),
$$

and it has a splitting as an $R$-module homomorphism. Thus $M_{i}$ is isomorphic to a direct summand of $\left(R^{1 / q}\right)^{\oplus r_{i}}$. This implies that $M_{i}$ is a direct summand of $R^{1 / q}$, since the category of $R$-modules is a Krull-Schmidt category.

Corollary 3.4. In the situation of Theorem 3.3, if we write $R^{1 / p^{e}}=\bigoplus_{i=1}^{n} M_{i}^{\oplus a_{i}^{(e)}}$, then the limit

$$
\lim _{e \rightarrow \infty} \frac{a_{i}^{(e)}}{p^{2 e}}
$$

exists and is a positive rational number for all $i=1, \ldots, n$.

\footnotetext{
${ }^{4}$ This monomorphism is exactly equal to the bidual of the map $M_{i}=M_{i} \otimes_{R} R \stackrel{c^{1 / q}}{\longrightarrow} M_{i} \otimes_{R} R^{1 / q}$.
} 
Proof. The existence and positivity of the limit follows from Smith-Van den Bergh [SVdB, Proposition 3.3.1] and the rationality from Seibert [Sei, Lemma 2.4].

Remark 3.5. (1) We note the difference between Corollary 3.4 and SVdB Proposition 3.3.1]. In our argument, $M_{1}, \ldots, M_{n}$ represent all isomorphism classes of indecomposable reflexive $R$-modules, whereas they are just those which appear as a direct summand of $R^{1 / p^{e}}$ for some $e \geq 0$ in [SVdB, Proposition 3.3.1].

(2) When a rational double point $R$ is a pure subring of $S=k[[x, y]]$, if we write $S=\bigoplus_{i=1}^{n} M_{i}^{\oplus b_{i}}$ as an $R$-module, then the above limit is described as

$$
\lim _{e \rightarrow \infty} \frac{a_{i}^{(e)}}{p^{2 e}}=\frac{b_{i}}{r} \in \frac{1}{r} \mathbb{Z}
$$

where $r=\sum_{i=1}^{s} b_{i} r_{i}=\operatorname{rank}_{R} S$; see [HS, Lemma 4.10].

To complete the proof of Theorem 3.1, we introduce some additional notation:

$\mu: \widetilde{X} \rightarrow X:$ the minimal resolution of $(X, x)$ with $\operatorname{Exc}(\mu)=\bigcup_{i=1}^{s} E_{i}$,

$\varphi=\varphi_{e}: \mathrm{FB}_{e}(X) \rightarrow X$ : the eth F-blowup.

Note that the F-regularity of $R=\mathcal{O}_{X}$ implies that $(X, x)$ is a rational singularity. Then by Wunram [W, Main Result (a)] 5 part of indecomposable reflexive $\mathcal{O}_{X}$-modules called special reflexives are in one-to-one correspondence with the irreducible exceptional curves $E_{1}, \ldots, E_{s}$ of the minimal resolution. We reorder the indecomposable reflexive $\mathcal{O}_{X}$-modules $M_{1}, \ldots, M_{n}$ so that the first $s$ of them, $M_{1}, \ldots, M_{s}(s \leq n)$, are special. Then one has

$$
c_{1}\left(\mu^{\star} M_{i}\right) E_{j}=\delta_{i j}
$$

for $1 \leq i, j \leq s$.

Now, with the aid of Wunram's result, the argument for F-rational double points in [HS, Proposition 4.9] works for F-regular surface singularities as well.

Proof of Theorem 3.1 continued. Let the reflexive $\mathcal{O}_{X}$-module $\mathcal{O}_{X}^{1 / p^{e}}$ be generated by $m$ elements and pick a surjection $\mathcal{O}_{X}^{\oplus m} \rightarrow \mathcal{O}_{X}^{1 / p^{e}}$. Since $\mu^{\star} \mathcal{O}_{X}^{1 / p^{e}}$ is a locally free $\mathcal{O}_{\widetilde{X}}$-module of rank $p^{2 e}([\mathrm{AV}])$, the induced surjection $\mathcal{O}_{\widetilde{X}}^{\oplus m} \rightarrow \mu^{\star} \mathcal{O}_{X}^{1 / p^{e}}$ gives rise to a morphism $\Phi_{e}: \widetilde{X} \rightarrow \mathbb{G}$ over $X$ to the Grassmannian $\mathbb{G}=\operatorname{Grass}\left(p^{2 e}, \mathcal{O}_{X}^{\oplus m}\right)$ such that $\mu^{\star} \mathcal{O}_{X}^{1 / p^{e}}$ is isomorphic to the pullback of the universal quotient bundle of $\mathbb{G}$.

Similarly, since the torsion-free pullback $\varphi^{\star} \mathcal{O}_{X}^{1 / p^{e}}$ to $Z=\mathrm{FB}_{e}(X)$ is locally free, the surjection $\mathcal{O}_{Z}^{\oplus m} \rightarrow \varphi^{\star} \mathcal{O}_{X}^{1 / p^{e}}$ gives rise to a morphism $Z=\mathrm{FB}_{e}(X) \rightarrow \mathbb{G}$ over $X$, through which $\Phi_{e}$ factors as

$$
\Phi_{e}: \widetilde{X} \rightarrow \mathrm{FB}_{e}(X) \rightarrow \mathbb{G}
$$

by Lemma 1.7. Composing $\Phi_{e}$ with the Plücker embedding $\mathbb{G} \hookrightarrow \mathbb{P}$ into the projective $N=\left(\left(\begin{array}{c}m \\ p^{2 e}\end{array}\right)-1\right)$-space $\mathbb{P}=\mathbb{P}_{X}^{N}$ over $X$, we obtain a morphism induced by the surjection $\mathcal{O}_{\widetilde{X}}^{\oplus N+1}=\bigwedge^{p^{2 e}} \mathcal{O}_{\widetilde{X}}^{\oplus m} \rightarrow \operatorname{det} \mu^{\star} \mathcal{O}_{X}^{1 / p^{e}}$, which coincides with the morphism

$$
\Phi_{|L|}: \tilde{X} \rightarrow \mathrm{FB}_{e}(X) \rightarrow \mathbb{P}
$$

given by the complete linear system associated to the $\mu$-generated line bundle $L=$ $c_{1}\left(\mu^{\star} \mathcal{O}_{X}^{1 / p^{e}}\right)$ by Lemma 1.8. Now if we write $F_{*}^{e} \mathcal{O}_{X}=\mathcal{O}_{X}^{\oplus a_{0}} \oplus \bigoplus_{i=1}^{n} M_{i}^{\oplus a_{i}^{(e)}}$, then

\footnotetext{
${ }^{5}$ Part (a) of the main results of $[\mathrm{W}]$ is verified to be true for rational surface singularities in arbitrary characteristic, without any change of the proof. See also AV.
} 
the intersection number of $L$ with each exceptional curve $E_{i}$ is $L \cdot E_{i} \geq a_{i}^{(e)}$ by (4), so that $L$ is $\mu$-very ample for $e \gg 0$ by Corollary 3.4. It follows that $\Phi_{e}$ is a closed immersion for $e \gg 0$ so that $\widetilde{X} \cong \mathrm{FB}_{e}(X)$.

Corollary 3.6. Let $(X, x)$ be an F-regular surface singularity and let $\pi:(S, o) \rightarrow$ $(X, x)$ be a finite covering from a smooth surface germ $(S, o)$ as in Theorem 2.1. Let $\operatorname{Hilb}_{d}(S / X)^{\circ}$ be the irreducible component of the relative Hilbert scheme $\operatorname{Hilb}_{d}(S / X)$ that dominates $X$, where $d=\operatorname{deg} \pi$. Then

$$
\widetilde{X} \cong \operatorname{Hilb}_{d}(S / X)^{\circ} \cong \mathrm{FB}_{e}(X)
$$

for $e \gg 0$.

Proof. Since $\mu^{\star} \pi_{*} \mathcal{O}_{S}$ is a locally free $\mathcal{O}_{\tilde{X}}$-module of rank $d$ ([AV]), we have a morphism $\tilde{X} \rightarrow \operatorname{Hilb}_{d}(S / X)^{\circ}$ over $X$. On the other hand, since the ring homomorphism $\mathcal{O}_{X} \rightarrow \pi_{*} \mathcal{O}_{S}$ splits as an $\mathcal{O}_{X}$-module homomorphism by Proposition 1.4 we have $\operatorname{Hilb}_{d}(S / X)^{\circ} \rightarrow \mathrm{FB}_{e}(X)$ by [HS, Proposition 4.2]. Thus the conclusion follows from Theorem 3.1 .

There exist non-F-regular surface singularities whose F-blowups are not smooth.

Example 3.7 ([HS, Example 4.4]). Let $X=\operatorname{Spec} k\left[\left[x^{p}, x^{p} y-x y^{p}, y^{p}\right]\right]$, where $k$ is a field of characteristic $p$, and let $g: X^{\prime} \rightarrow X$ be the weighted blowup. The exceptional set of $g$ is a single $\mathbb{P}^{1}$, on which $X^{\prime}$ has $p+1 A_{p-1}$-singularities. Resolving these $A_{p-1}$-singularities, we obtain the minimal resolution $f: \widetilde{X} \rightarrow X$. (So in case $p=2, X$ has a rational double point of type $D_{4}$.) It follows that the torsion-free pullback $g^{\star} \mathcal{O}_{X}^{1 / p^{e}}$ is a flat $\mathcal{O}_{X^{\prime}}$-module so that $\mathrm{FB}_{e}(X)$ is dominated by $X^{\prime}$ for all $e \geq 0$. Thus the F-blowups of $X$ do not coincide with any resolution of $X$.

The behavior of F-blowups for non-F-regular surface singularities is a mystery yet. We pose here two extremal questions in opposite directions.

Questions. (1) Let $(X, x)$ be a log terminal surface singularity in characteristic $p>0$. Does $\mathrm{FB}_{e}(X)$ coincide with the minimal resolution of $X$ for $e \gg 0$ only if $(X, x)$ is F-regular?

(2) Let $(X, x)$ be a normal surface singularity defined over $\mathbb{Q}$. Does the Fblowup of reduction $\left(X_{p}, x_{p}\right)$ modulo $p$ of $(X, x)$ coincide with the minimal resolution for $p \gg 0$ (or infinitely many $p$ )?

\section{ACKNOWLEDGEMENTS}

The author is grateful to Tomohiro Okuma for pointing out that a proof for Theorem 2.1 is given in BHPV] and to Noboru Nakayama for showing another proof given in his preprint $[\mathrm{LN}]$ with Y. Lee.

\section{REFERENCES}

[Ar1] Artin, M., Some numerical criteria for contractability of curves on algebraic surfaces, Amer. J. Math. 84 (1962), 485-496. MR0146182(26:3704)

[Ar2] Artin, M., Covering of the rational double points in characteristic $p$, Complex Analysis and Algebraic Geometry, pp. 11-22, Iwanami Shoten, Tokyo, 1977. MR0450263 (56:8559)

[AV] Artin, M. and Verdier, J.-L., Reflexive sheaves over rational double points, Math. Ann. 270 (1985), 79-82. MR769609 (85m:14006)

[Aus] Auslander, M., Rational singularities and almost splitting sequences, Trans. Amer. Math. Soc. 293 (1986), 511-531. MR.816307 (87e:16073) 
[BHPV] Barth, W. P., Hulek, K., Peters, C. A. M., and Van de Ven, A., Compact Complex Surfaces (second edition), Ergebnisse der Mathematik und ihrer Grenzgebiete, Folge 3, 4, Springer-Verlag, Berlin, 2004. MR2030225 (2004m:14070)

[GSV] Gonzalez-Sprinberg, G., and Verdier, J.-L., Construction géométrique de la correspondance de McKay, Ann. Sci. Ec. Norm. Sup. $4^{e}$ Sér. 16 (1983), 409-449. MR740077 (85k:14019)

[H] Hara, N., Classification of two-dimensional F-regular and F-pure singularities, Adv. Math. 133 (1998), 33-53. MR 1492785 (99a:14048)

[HS] Hara, N. and Sawada, T., Splitting of Frobenius sandwiches, RIMS Kôkyûroku Bessatsu B24 (2011), 121-141.

[HW] Hara, N. and Watanabe, K.-i., F-regular and F-pure rings vs. log terminal and log canonical singularities, J. Algebraic Geometry 11 (2002), 363-392. MR.1874118(2002k:13009)

[HH] Hochster, M. and Huneke, C., Tight closure and strong F-regularity, Mem. Soc. Math. France 38 (1989), 119-133. MR1044348 (91i:13025)

[Hu] Huneke, C., Tight Closure and Its Applications, CBMS Regional Conference Series in Mathematics, Number 88, American Mathematical Society, 1996. MR.1377268 (96m:13001)

[I] Ishii, A., On the McKay correspondence for a finite small subgroup of $G L(2, \mathbb{C})$, J. Reine Angew. Math. 549 (2002), 221-233. MR.1916656 (2003d:14021)

[K] Kawamata, Y., Index 1 covers of log terminal surface singularities, J. Algebraic Geom. 8 (1999), 519-527. MR:1689354 (2001f:14066)

[LN] Lee, Y. and Nakayama, N., Simply connected surfaces of general type in positive characteristic via deformation theory, preprint.

[Si] Singh, A. K., $\mathbb{Q}$-Gorenstein splinter rings of characteristic $p$ are F-regular, Math. Proc. Cambridge Philos. Soc. 127 (1999), 201-205. MR1735920 (2000j:13006)

[Sei] Seibert, G., The Hilbert-Kunz function of rings of finite Cohen-Macaulay type, Arch. Math. (Basel) 69 (1997), 286-296. MR 1466822 (98h:13022)

[SVdB] Smith, K. E. and Van den Bergh, M., Simplicity of rings of differential operators in prime characteristic, Proc. London Math. Soc. (3) 75 (1997), 32-62. MR1444312 (98d:16039)

[TY] Toda, Y. and Yasuda, T., Noncommutative resolution, $F$-blowups and $D$-modules, $A d v$. Math. 222 (2009), 318-330. MR:2531377 (2011b:14005)

[W] Wunram, J., Reflexive modules on quotient surface singularities, Math. Ann. 279 (1988), 583-598. MR926422 (89g:14029)

[Y1] Yasuda, T., Universal flattening of Frobenius, to appear in the Proceedings of the 5th Franco-Japanese symposium on singularities, 2009.

[Y2] Yasuda, T., On monotonicity of F-blowup sequences, Illinois J. Math. 53 (2009), 101110. MR 2584937 (2011b:14007)

Mathematical Institute, Tohoku University, Sendai 980-8578, Japan

E-mail address: hara@math.tohoku.ac.jp 